\title{
Reflections on the Definition of "Art" in George Dickie's Theory of Art Convention
}

\author{
Wang Junying ${ }^{1, ~ a ~}$ \\ ${ }^{1}$ Dalian Polytechnic University, Ganjingzi, Dalian, Liaoning, China \\ a747086535@qq.com

\begin{abstract}
George Dickie, an American Analytical aesthetician, makes a new exposition of art from the perspective of the process or steps that a thing must go through to become a work of art. To some extent, this definition reflects the important role of art mechanism in contemporary society and art circles, and at the same time, there are many internal contradictions in the definition itself.
\end{abstract}

Keywords: Artistic conventions, artifact, art world, award

\section{关于乔治・迪基艺术惯例说中“艺术”定义的思考}

王珺英 $1, \mathrm{a}$

\author{
1 大连工业大学, 甘井子, 大连, 辽宁, 中国 \\ a747086535@qq.com
}

\section{摘要}

美国分析美学家乔治・迪基从一个事物要成为艺术作品所必须经历的过程或步骤的角度对艺术进行了新的阐述。 这一定义一定程度上反映了艺术机制在当代社会与艺术界中的重要作用, 而同时该定义本身又存在很多内在 矛盾。

关键词: 艺术惯例人工制品 艺术界授予

\section{1.前言}

关于何为 “艺术”, 在古希腊时期, 就引发了哲学 家们及艺术家们的探讨。而从 18 世纪现代艺术体系 开始逐渐被确立以来, 对艺术本质的研究, 更是众说 纷纭。譬如: 德国哲学家康德认为 “艺术是某个事物 美的表象, 是建立在人理性基础上的自由的创造活 动”; 其学生黑格尔则提出 “艺术无论是目的还是手 段, 都是自由的艺术”; 克罗齐则认为 “艺术即直觉”; 科林伍德提出 “艺术即想象”; 克莱夫 . 贝尔提出 “艺 术是有意味的形式”; 杜威指出“艺术即经验”; 恩斯特 . 卡西尔认为 “艺术是一种符号语言”; 苏珊. 朗格 进一步发展了卡西尔的观点, 认为 “艺术是人类情感 的符号”; 托马斯 . 门罗又提出了 “艺术作品是人类 技艺的产品” 等等众多“艺术”的定义。这些五花八门 的定义, 皆在相对历史阶段中具有一定的合理性, 而 由于艺术实践的不断发展, 艺术的内涵也在不断的发 生变化。因而, 可以说艺术是一个历史性的概念, 其
产生以后，不断地发展演进，不断地发生变异。“艺 术” 概念本身的复杂性与变异性, 势必会使其难以定 义。而从这些形形色色的传统艺术定义中, 至少有两 点共同性: 其一, 基本上都承认艺术是一种技术; 其 二, 大多都承认艺术基于审美经验。直到 20 世纪, 摄影的发展和电脑技术的产生, 使得艺术家的技术受 到冲击; “同时艺术创作是否基于美感经验也值得怀 疑” ${ }^{[1]}$, 以至于传统的艺术定义中最后两个共性似乎 也不复存在了。而当杜尚把一个实用的小便池, 拿到 美术馆去，作为一件艺术作品以后，艺术与非艺术之 间更难以区分。

虽然, 艺术的概念难以定义，但是美学家和艺术 批评家仍在不断的探索, 希望能找到一个“放之四海” 而皆被认同的概念。从二十世纪六十年代以来出现的 艺术定义来看, 大致可以分为两类: “程序性的定义 和功能性的定义。功能性的定义是从艺术作为一种创 造性的产品究竟要实现怎样的目的或发挥怎样的功 能上来进行定义。所有的传统的艺术定义都属于功能性 
的定义。程序性的定义是从一个东西要成为艺术作品 所必须经历的过程或步骤上来进行定义的。” ${ }^{[2]}$ 美国 当代著名哲学家、美学家、后期分析美学的主要代表 乔治・迪基认为，任何从功能方面对艺术的定义都是 不可能成功的; 同时他肯定艺术的本质是可以把握的, 对艺术下定义也是可能的。基于对其他美学流派和美 学理论思想兼收并蓄, 乔治. 迪基尝试探索一种新的 途径来给艺术定义, 即从程序方面来对艺术的定义进 行把握。

\section{2. 乔治・迪基艺术惯例说的理论基础}

与前期分析美学家不同, 乔治. 迪基认为艺术是 可以下定义的, 艺术的本质也是可以把握的。迪基对 于其他美学流派和美学理论思想兼收并蓄, 用折衷主 义的态度, 提出了著名的习俗论美学, 试图将传统美 学和分析美学进行调和和折衰, 进而习俗论表现出一 种与分析美学若即若离的倾向。

从整体上看, 一方面, 乔治. 迪基对于传统美学 的主导态度是批判的。首先, 他对康德以来从审美无 利害关系态度论进行了深入的批判; 其次, 他对传统 美学中两种影响最大的美学理论一一模仿论和表现 论进行批判。他认为, 模仿说注重艺术与题材的关系, 表现说则注重作品与它的创作者之间的关系, 他们 “只看到艺术作品那些突出的但是偶然的特性, 而不 能发现那些显示艺术在历史发展中某一阶段的特 性。” ${ }^{[3]}$ 另一方面, 乔治. 迪基从当代美学家丹托、 曼德尔鲍姆、大卫・刘易斯那里得到了有益的启发。 丹托在《艺术世界》一文中指出了 “艺术界（art world)” 这一重要概念。丹托认为一个人是否成为艺 术家, 一件作品能否成为艺术品, 这是由 “艺术界” 及其成员来确定的。“把某物看作艺术, 需要眼睛不 能察觉的东西一一一种艺术理论的氛围, 一种艺术史 的知识: 一个艺术界。” ${ }^{[4]}$ 丹托从艺术作品的非外现 的特性方面来规定艺术的本质, “艺术界” 正是这样 一些特性的总和, 它涉及了艺术的环境氛围、理论和 历史。丹托同时提出艺术界中的艺术理论乃是区分艺 术与非艺术的根本尺度, 即 “艺术理论倡导的特定习 俗有可能成为艺术。” ${ }^{[5]}$ 迪基认为丹托指出了艺术的 习俗性质, 并用丹托的 “艺术界” 这一概念来指代广 泛的社会习俗。另一位分析美学家曼德尔鲍姆在指责 维特根斯坦强调 “可以直接显现出来的类似性” 时, 指出艺术还有非外现的特性。乔治. 迪基从曼德尔鲍 姆关于艺术非外现的观点中受到启发, 认为只要能把 握非外现特性, 就能抓住艺术品共同的东西, 就能避 免像传统美学那样仅抓住外现特征为艺术下定义的 局限。美国哲学家、美学家刘易斯在迪基之前提出了 他自己的 “艺术习俗论”, 认为习俗是一种默契, 一种 协调和平衡，艺术则与这种平衡达到了奇异的结合。 在迪基酝酿习俗论时, 刘易斯的这种习俗观点也对他
产生了重要的影响。可以说, 乔治. 迪基的习俗论是 在批判、继承和发展中逐渐产生的。

\section{3. 乔治・迪基对艺术的定义}

早先乔治．迪基对艺术的定义是从分类意义上 的艺术品而言的。他认为 “艺术品” 至少有三种截然 不同的意义: 基本的或分类的意义、次要的或派生的 意义、评价意义。其中, 评价意义是根据性质来定义 的, 不考虑所指对象本身是什么, 只从欣赏角度来评 价。基本的或分类的意义是决定一对象是不是一件艺 术品的基础。迪基指出，艺术品的分类意义主要有两 点: 一是人造性; 另一是习俗性, 二者都是艺术品的 非外现特性。这里迪基所谓的 “习俗” 是一种已被建 立的惯例。在艺术的历史发展中已经形成种种艺术习 俗, 它作为艺术品得以存在的构架而存在。迪基习俗 论的核心是 “艺术界”。他认为 “习俗是由艺术世界的 实践活动来决定的，艺术世界的工作在一种常规的实 践层次上进行, 它仍然是一种实践活动, 通过这种活 动, 制定出一种社会习俗。” ${ }^{[6]}$ 作为习俗的 “艺术界” 既包括种种约定俗成的惯例, 这些惯例组成了戏剧、 绘画等不同的艺术子系统，众多艺术子系统又构成了 艺术系统; 艺术界也包含了艺术家、艺术观众、艺术 批评家等在内的一切艺术活动的参加者, 他们使得艺 术界保持运行。迪基进一步指出: 艺术界的根本作用 是将艺术地位授予对象提供一种习俗背景。显然, 这 里提及的 “艺术界” 深受似丹托 “艺术界” 的影响。 进而, 乔治. 迪基认为: 要确定一对象究竟是否艺术 品, 要看作为习俗的艺术界是否授予其作为艺术品的 地位。类似于国王授予某人以爵位，在艺术领域中， 艺术品的地位也是在艺术界的框架内被授予的。

如此，乔治．迪基给艺术下的定义为: “一个艺 术品在它的分类意义上是：1. 一个人工制品，2. 代表 某种社会制度（艺术界）的一个人或一些人授予它具 有欣赏候选资格的地位。” ${ }^{[7]}$ 可以看出, 这个定义与 艺术作品的目的毫无关系，它并不涉及审美经验等问 题。而是根据程序的方法, 即一对象成为艺术作品经 历的程序 (过程), 从对象的非外现特性方面给艺术 定义的。在这个艺术定义里, 迪基首先强调了艺术的 社会文化性。当迪基用社会制度来规定艺术时，也就 是把艺术置于社会文化的大背景中考虑。其次，迪基 注重从艺术家和艺术欣赏者的统一中认识艺术本质 问题。可见，乔治．迪基的艺术惯例说在美学上是有 着一定的积极意义和突破性的。

\section{4. 对乔治 · 迪基艺术定义的思考}

乔治 . 迪基提出的艺术惯例说，是从程序角度考 察艺术，在分析美学中产生了巨大的影响，同时也在 某种程度上反映了当代西方国家艺术的运行制度。而 其中当然，迪基的艺术定义也在很多方面面临着质疑。 


\section{1. 人工制品的范围到底有多宽泛}

按照乔治 · 迪基艺术惯例说中对艺术的定义, 一 对象要成为艺术品首先应当是一件 “人工制品”。但 是, 以杜尚《泉》为代表的“现成制品”, 和像 “浮木” 一样的自然物, 以及猩猩等乱涂的动物画, 也都被迪 基授予了艺术品的地位。其中, 迪基曾以杜尚的《泉》

(图 1) 为例做过详细的阐述, 来说明现成品也能成 为艺术品。他认为 “作为一件实用品的小便藤之所以 是艺术品, 那是因为一位艺术家一一杜尚授予了它以 艺术品的地位”。 ${ }^{\left[{ }^{[8]}\right.}$ 显然, 迪基所说的人工性不是仅仅 产生于加工创作, 而是还包含 “授予” 在内。这就改 变了以往只有绘画、雕塑或其他富含手工技艺的作品, 才能被视为艺术的观念。可见, 迪基所提出的这种“授 予” 是对一切传统艺术观念彻底的否定与批判, 同时 在理论上给了现代派艺术以广泛的空间。按照迪基的 理论, 杜尚的另一作品《带胡须的蒙娜丽莎》自然也 被认可为艺术作品了。该作品为杜尚在 1917 年, 把 达 - 芬奇举世闻名的《蒙娜丽莎》的一件印刷品上, 用铅笔涂上了山羊胡子, 原本蒙娜丽莎的神秘微笑, 立即消失殁尽, 画面一下子变得稀奇而古怪, 并标有 “L. H. 0. 0. Q” 的字样 (图 2)。《蒙娜丽莎》的印刷品 无疑属于人工制品, 同时杜尚也的确在这件印刷品上 进行了画胡子的创作, 因而该作品完全满足了迪基对 艺术的限定条件。然而, 当下模仿《带胡须的蒙娜丽 莎》的一些恶搞, 如凤姐、小胖等网红利用软件技术 将《蒙娜丽莎》置换成自己的头像 (图 3)。这种简单 替换的恶搞, 按照迪基所持的观点, 也是满足了艺术 作品的条件的, 也是可以被视为艺术, 岂不荒诞! 而 如此的话, 艺术与非艺术的界限变得更加模糊, 艺术 家与普通人甚至也没有区别。可见, 迪基从程序的角 度为艺术下的这个定义, 对人工制品的限定范围十分 宽容, 企图囊括当前的前卫艺术与先锋艺术。

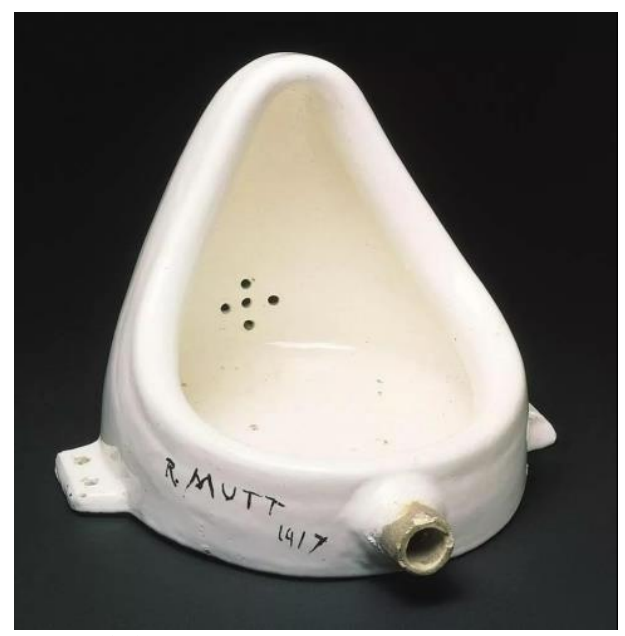

图 1 杜尚, 《泉》. 1917 年

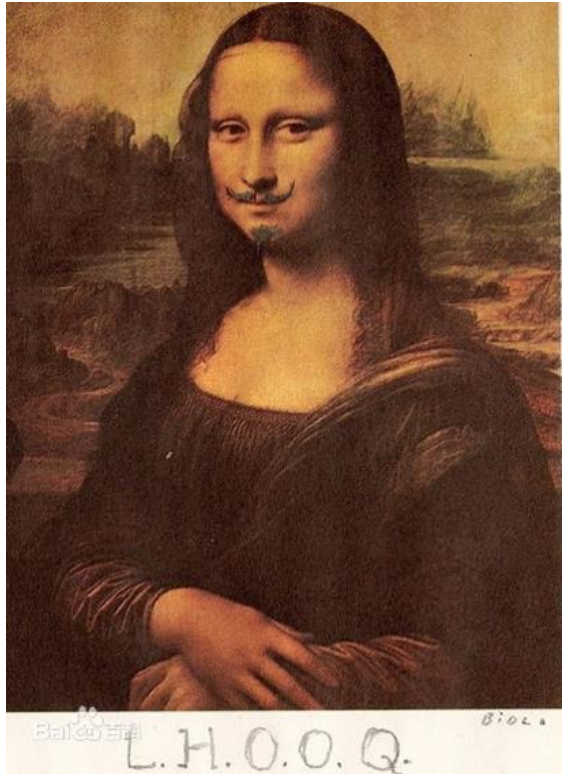

图 2 杜尚, 《带胡须的蒙娜丽莎》. 1917 年
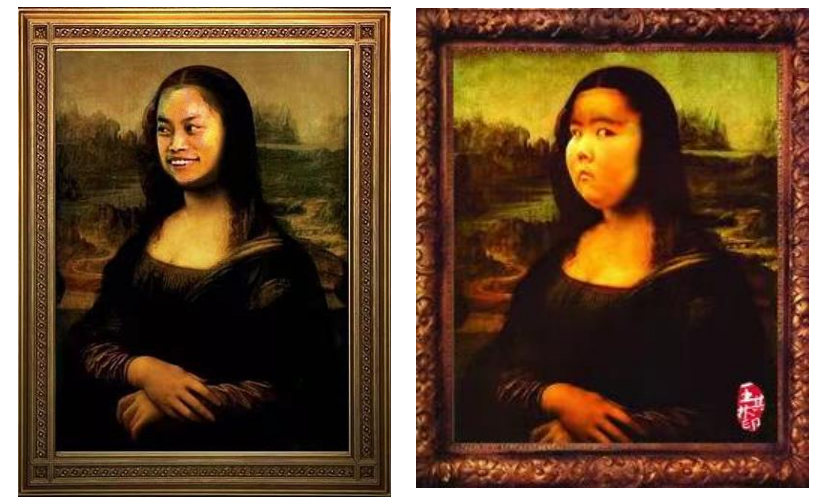

图 3 网红恶搞蒙娜丽莎

按照迪基的这种人工制品是 “授予” 的观点, 一 块 “浮木”成为艺术品也不难理解。“浮木” 虽然是自 然物, 但是只要它被捡起, 带回家或挂在墙上或送交 某个展览馆, 这些过程的完成就已经有人的活动参与 其中。“浮木” 被人带到了艺术界中, 有了人的因素, 所以 “浮木” 是人工制品的观点也是成立的。另外, 人在选择 “浮木” 时, 面临了选择 “此浮木” 还是 “彼 浮木”, 选择 “浮木” 还是 “石头” ......多种思考来篮 选自然物对象, 这个过程也是人的一种意识活动。所 以, 对于被选择成为艺术品的自然物来说, 无论从人 的具体物质活动, 还是从人的抽象意识活动上说自然 物都可以称为人工制品。

如果说，现成制品和自然物可以通过 “授予” 成 为人工制品的话, 那么动物画的 “人造性” 又从何而 来? 迪基把猩猩乱涂的动物画（图 4) 也看成是艺术 品。如果按照以上论述自然物和现成品的 “人造性” 那样照葫芦画㼼, 那么猩猩乱涂 “将成为负责展出这 些画的个人的艺术” [9]艺术家将猩猩的乱涂冠以自己 的名义展出, 岂不滑稽! 可见, 迪基的艺术定义企图 包容所有艺术作品, 对任何艺术都采取了极为宽容的 态度, 这就使得迪基的艺术定义有无限的任意性, 最 终势必陷于矛盾之中。 


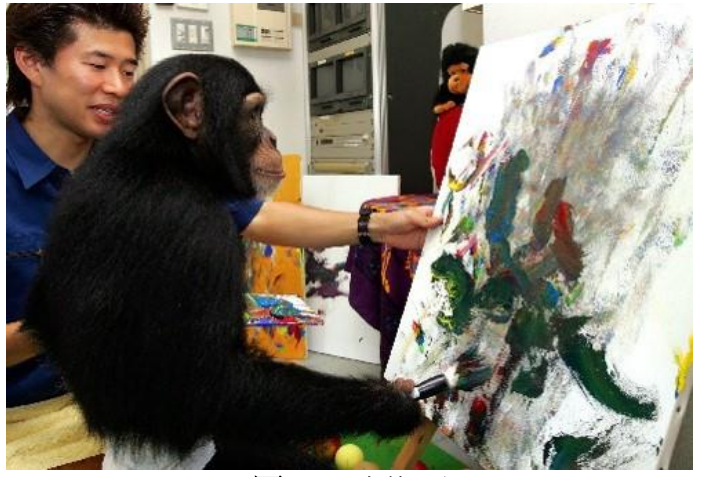

图 4 动物画

\section{2. 当 “个人授予”与 “公众授予” 矛盾时 应如何界定}

当对于同一人工制品, 它的创造者承认它是艺术 品, 而公众也支持同一看法时, 那么不会产生疑惑; 但当公众并不承认它是艺术品时或是公众中的部分 认为它是艺术品, 它又是不是艺术呢? 迪基曾指出 “授予” 活动既可以由包括艺术批评家、艺术欣赏者 在内的一大批人来进行, 也可以由一个人（艺术家自 己）来进行。迪基的 “授予” 说既顾及到公众对于艺 术品的普遍承认, 又强调艺术家或艺术界的一个人对 于艺术品的单一认同。虽然杜尚认为小便池是艺术品, 但是杜尚在选择小便壸时, 他的头脑中想的自然是这 个小便壸能否被博物馆接受的问题。倘使被博物馆接 受, 那么小便壶就成为一件艺术品, 否则亦然。所以, 在艺术体制健全的西方社会中, 个人授予似乎最终还 是直于艺术机制的限制。在迪基的艺术定义里, 当“个 人授予” 与 “公众授予” 不协调时应如何解决, 似乎 迪基并没有化解这个矛盾。

\section{3. 候选资格的尺度问题}

按照迪基的观点某人或某些人代表某个制度来 确定艺术作品的欣赏对象资格, 那么这个资格是怎样 确定的？他或者他们根据什么确定这个人工制品具 有候选欣赏资格而另一个则不具有, 衡量的尺度是什 么? “显然, 迪基将什么是艺术的问题, 隐含到或者 转换为什么是艺术的候选资格问题。这种隐含或转让 具有极大的欺骗性。表面看来它似乎解决了决定什么 是艺术的核心问题，事实上它只不过将这个问题推延 给了艺术界, 让艺术界去决定, 至于艺术界究竟怎么 决定, 似乎就成了另外一个跟艺术定义无关的问题 了。” $[10]$

\section{4. 自由与保守的矛盾}

迪基的艺术定义中所说的 “授予” 活动是发生在 “艺术界” 中的。“艺术界” 的实质就是一种习俗。作 为“艺术界”它是由惯例和被习俗化了的人构成的。可 见, 习俗是保守的, 作为一种约定俗成的默契与平衡, 它的社会性根基是难以被动摇的。但是, 艺术却总是 包含着创造性、变化性和自由性的因素。保守的习俗
与自由创造的艺术之间必然存在着诸多隔阂。保守与 自由应如何调和? 用约定俗成的习俗来规范变化的 艺术，势必会面临捉襟见肘的问题。

\section{5. 结语}

乔治 ·迪基艺术惯例说中，首次从程序的角度指 出, 一个艺术品在它的分类意义上是：1.一个人工制 品, 2.代表某种社会制度 (艺术界) 的一个人或一些 人授予它具有欣赏候选资格的地位。而后, 乔治. 迪 基又对该定义进行了进一步的修定，认为艺术必须是 件人工制品，而且它是为提交给艺术界的公众而创造 出来的。在早晚期两个版本的艺术定义中, 本文中涉 及的质疑, 并没有得到根本的解决。尽管如此, 不影 响该理论在美学上的地位。特别是它反映了现代社会, 尤其是在艺术体制非常健全的西方国家里, 艺术机构 或者体制是凌驾于艺术家和公众之上的最终主宰者。 实际上, 迪基的艺术定义揭示了现代社会中的艺术规 则。而在艺术惯例说基础上，乔治 . 迪基提出的 “发 现” 概念与 “约定” 概念更有价值和启发。迪基说: “金” 的概念的确立是发现的一一化学家通过科学分 析, 对原子序数为 79 的化学元素称为“金”。化学家 对“金”的定义是放之四海而皆准，所以，“金”的概念 很快的且很容易的被传播了。而“单身汉”的概念的确 立是约定的一一不同地域、不同时代、不同国家等对 于“单身汉”的理解不同。比如, 未婚的男子、丧妻的 男子、无子的男子以及教士都可以被称为“单身汉”。 单身汉”概念的差异使得它的传播是很困难的。迪基 认为, “艺术” 的概念类似于 “单身汉” 的概念, 它们 都是约定的概念。所以，没有关于 “金” 的概念的论 争，却有关于 “艺术” 概念的争论，才会有关于艺术 的哲学。正如迪基有关 “术的定义存在着某些缺陷, 引起许多争议，但仍有许多值得重视的地方。艺术的 定义、艺术的哲学正是在不断的争论和批判中发展和 丰富的。

\section{项目基金}

本文为 2021 年辽宁省社会科学规划基金 重点项目《辽宁省民间手工艺的保护与振兴研 究》(L21AMZ002)的阶段性成果之一。

\section{REFERENCES}

[1] Peng Feng,P.(2005)Western Aesthetics and art.Peking University Press, Beijing.204-210.

[2] Zhu Liyuan,Z. Zhang Dexing,Z. etc.(1999) General history of western aesthetics $\bullet$ aesthetics in the 20th century (top and bottom), Shanghai Literature and Art Publishing House.166-173.

[3] Jiang Kongyang,J.(1999)Selected works of Western Aesthetics in the 20th century (Part 2),Fudan University Press, 126-143. 
[4] Chi Yu Chen, C.(2005)Introduction to modern art.

Tsinghua University Press,26-27.

[5] Peng Feng,P.(2001) From analytical philosophy to Pragmatism -- a new direction of contemporary western aesthetics,Foreign social sciences, 2001 (4), $37-43$.

[6] George Dickie,G.Liu YueDi,L. (2015)"Art convention theory": early and late versions, Journal of Yantai University (PHILOSOPHY AND SOCIAL SCIENCES EDITION) 2015,28(02).

[7] (US) edited by M. Lippmann, translated by Deng Peng. Contemporary aesthetics, Guangming Daily Press, 1986. 